Case Report

\title{
Pseudotumor Cerebri in a Child with Idiopathic Growth Hormone Insufficiency Two Months after Initiation of Recombinant Human Growth Hormone Treatment
}

\author{
Eleni Loukianou, ${ }^{1}$ Anastasia Tasiopoulou, ${ }^{1}$ \\ Constantinos Demosthenous, ${ }^{1}$ and Dimitrios Brouzas ${ }^{2}$ \\ ${ }^{1}$ Department of Ophthalmology, Makarios Hospital, 2012 Nicosia, Cyprus \\ ${ }^{2} 1$ st University Eye Clinic, 11527 Athens, Greece \\ Correspondence should be addressed to Eleni Loukianou; eleniloukianou@yahoo.com
}

Received 24 November 2015; Revised 17 January 2016; Accepted 20 January 2016

Academic Editor: Ruth Huna-Baron

Copyright (c) 2016 Eleni Loukianou et al. This is an open access article distributed under the Creative Commons Attribution License, which permits unrestricted use, distribution, and reproduction in any medium, provided the original work is properly cited.

Purpose. To report a rare case of pseudotumor cerebri (PTC) in a child two months after receiving treatment with recombinant human growth hormone (rhGH) and to emphasize the need of close collaboration between ophthalmologists and pediatric endocrinologists in monitoring children receiving rhGH. Methods. A 12-year-old boy with congenital hypothyroidism started treatment with $\mathrm{rhGH}$ on a dose of $1,5 \mathrm{mg}$ /daily IM (4.5 IU daily). Eight weeks later, he was complaining of severe headache without any other accompanying symptoms. The child was further investigated with computed tomography scan and lumbar puncture. Results. Computed tomography scan showed normal ventricular size and lumbar puncture revealed an elevated opening pressure of $360 \mathrm{~mm} \mathrm{H}_{2} \mathrm{O}$. RhGH was discontinued and acetazolamide $250 \mathrm{mg}$ per os twice daily was initiated. Eight weeks later, the papilledema was resolved. Conclusions. There appears to be a causal relationship between the initiation of treatment with rhGH and the development of PTC. All children receiving rhGH should have a complete ophthalmological examination if they report headache or visual disturbances shortly after the treatment. Discontinuation of rhGH and initiation of treatment with acetazolamide may be needed and regular follow-up examinations by an ophthalmologist should be recommended.

\section{Introduction}

Pseudotumor cerebri is a disorder defined as an elevated intracranial pressure with normal cerebrospinal fluid composition documented by lumbar puncture and normal neuroimaging with absence of deformity, displacement, or obstruction of the ventricular system documented by computed tomography and magnetic resonance imaging. It is more common in adults and the diagnostic criteria of PTC in this age group are summarized in Box 1 [1-3].

It can also occur at any age in childhood. It is reported that $60 \%$ of children developing PTC are over the age of ten years [4]. As PTC in young children is different in clinical presentation from adolescents and adults new diagnostic criteria for PTC in prepubertal children were established (Box 2) [5].
Usual presenting symptoms in children include headache, nausea, vomiting, unilateral or bilateral transient obscurations lasting seconds, pulsatile tinnitus, double vision, blurred vision, and stiff neck [4]. In adolescents the most common presenting complaint is headache, whereas in younger children and infants it is irritability rather than headache. Headache may be throbbing and wake the patient from sleep [6, 7]. In pediatric population, PTC is associated with endocrine abnormalities, medications, viral infections, nutritional etiologies, or systemic conditions as shown in Box $3[6]$.

Recombinant human growth hormone was first introduced in medical practice in 1985 [8, 9]. It is produced through recombinant DNA technology and has primarily been used in the treatment of children with idiopathic growth hormone deficiency. It has also been used to treat other 
(1) Signs and symptoms of increased intracranial pressure

(2) No localizing neurological signs

(3) Normal MRI/CT brain scans with no evidence of central venous sinus thrombosis

(4) Increased intracranial pressure over $250 \mathrm{~mm} \mathrm{H}_{2} \mathrm{O}$ and normal cerebrospinal fluid composition

(5) No other identified cause of intracranial hypertension

Box 1: Diagnostic criteria for PC in adults.

(1) Symptoms or signs of generalized intracranial hypertension or papilledema with normal mental status

(2) Documented elevated intracranial pressure (age appropriate) measured in the lateral decubitus position; neonates: $>76 \mathrm{~mm} \mathrm{H}_{2} \mathrm{O}$, age 1-18 years: $>280 \mathrm{~mm} \mathrm{H}_{2} \mathrm{O}$

(3) Normal CSF composition except in neonates who may have up to $19 \mathrm{WBC} / \mathrm{mm}^{3}$ if $0-28$ days and up to $9 \mathrm{WBC} / \mathrm{mm}^{3}$ if between 29 and 56 days of age; the protein may be as high as $150 \mathrm{mg} / \mathrm{dL}$

(4) No evidence of hydrocephalus, mass, structural, or vascular lesion on MRI,

with and without contrast, and MR venography; narrowing of the transverse sinuses is allowed

(5) Cranial nerve palsies without any other identifiable etiology which improve with reduction of

cerebrospinal fluid pressure or resolution of other signs and symptoms of intracranial hypertension

(6) No other identified cause of intracranial hypertension

Box 2: Diagnostic criteria for prepubertal PTC.

(1) Endocrine abnormalities (thyroid disease, Addison disease, corticosteroid withdrawal, treatment with rhGH, desmopressin nasal spray, levonorgestrel implants)

(2) Medications (nalidixic acid, tetracyclines, nitrofurantoin, chemotherapies)

(3) Viral infections (varicella, measles)

(4) Nutritional etiologies (vitamin A toxicity, vitamin A or D deficiencies)

(5) Systemic conditions (Miller-Fisher syndrome, acute lymphocytic leukemia, Turner syndrome, galactosemia, galactokinase deficiency)

Box 3: Etiology of PTC in children.

conditions including Turner syndrome, delayed puberty, and empty sella syndrome [8]. Many cases of PTC were reported in children treated with rhGH since 1993 [10]. The prevalence of PTC in pediatric population receiving treatment with rhGH is approximately one hundred times greater than in the normal population [11].

\section{Case Presentation}

A 12-year-old boy presented at the Paediatric Department complaining of severe headache without any other symptoms. He had a past medical history of congenital hypothyroidism and idiopathic growth hormone insufficiency. His body mass index (BMI) was 13 and he was under thyroxine treatment. He was also on the 8th week of recombinant human growth hormone $(\mathrm{rhGH})$ treatment on a dose of $1.5 \mathrm{mg}$ daily (or $4.5 \mathrm{IU}$ daily). On the same day he was referred to the Eye Department for further assessment.

On initial examination his best corrected visual acuity was $6 / 9$ in both eyes. His intraocular pressures (IOPs) were $12 \mathrm{mmHg}$ in the right eye and $15 \mathrm{mmHh}$ in the left. Color vision tested via Ishihara plates showed no defects in either eye. Moreover, the anterior chamber and the papillary reflexes were normal and a relative afferent papillary defect (RAPD) was not revealed in either eye. Fundoscopy revealed bilateral hyperaemic optic nerve edema, with no spontaneous venous pulsations and with increased retinal vessel tortuosity. Elevation of nasal and temporal circumference of the optic nerve obscuring some segments of the major retinal vessels and a halo surrounding the disc were apparent in both eyes (Stage 3, staging scheme of swelling of the optic nerve head by Frisen) [12] (Figures 1(a) and 1(b)).

Visual field testing was inconclusive because the patient was uncooperative. There was a second effort of visual field testing which showed an enlargement of the blind spot in both eyes without any other abnormalities. Further investigations were performed including blood pressure measurements and blood tests which were within normal limits. An assessment by a neurologist took place and a brain computed tomography (CT) scan was ordered. The CT scan showed normal ventricular size, swelling of optic nerve heads, and a partially empty sella without any evidence of mass, haemorrhage, edema, or midline shift. Lumbar puncture revealed an elevated opening pressure of $36 \mathrm{~cm}$ water and a normal spinal fluid composition. The diagnosis of pseudotumor cerebri was established and the young patient 


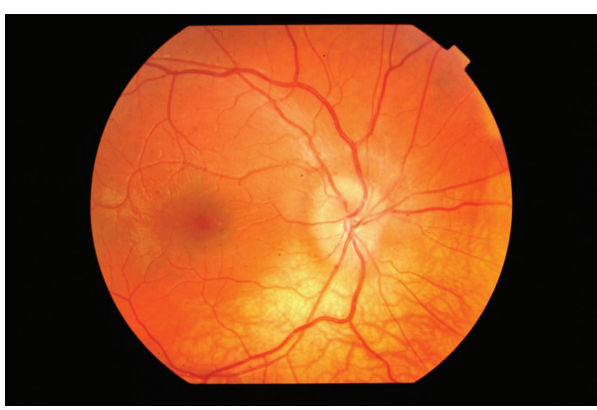

(a)

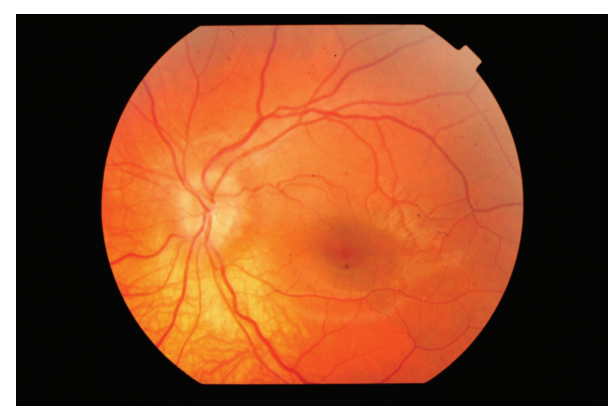

(b)

FIGURE 1: ((a), (b)) Bilateral optic disc edema on initial examination. There is elevation of nasal and temporal circumference of the optic nerve with a halo surrounding the disc completely.

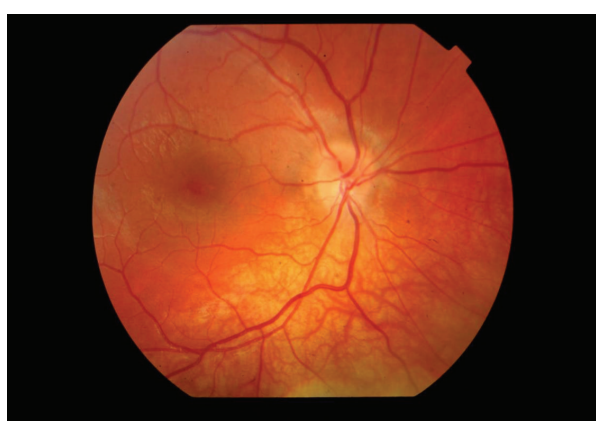

(a)

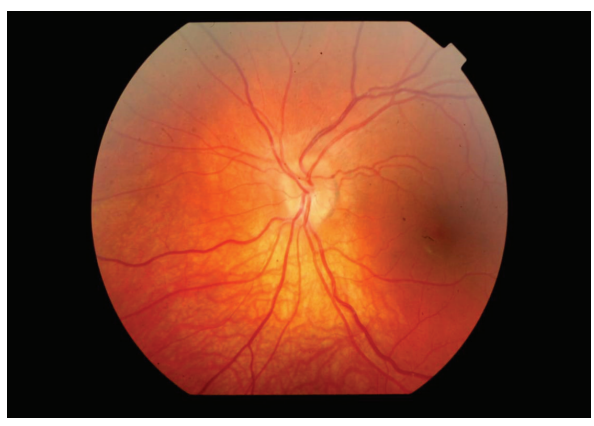

(b)

Figure 2: ((a), (b)) Partial resolution of right and left optic nerve edema 4 weeks after discontinuation of rhGH.

was admitted to the hospital. rhGH was discontinued and a treatment with acetazolamide ( $250 \mathrm{mg}$ per os twice daily) was initiated.

The patient was reviewed regularly. Four weeks later, his best corrected visual acuity was 6/9 in both eyes. His intraocular pressures and color vision perception were normal. Fundoscopy revealed partial resolution of optic nerve edema in both eyes (Figures 2(a) and 2(b)).

Visual field testing was repeated showing blind spot enlargement in both eyes. After discussion with the pediatric endocrinologist acetazolamide was increased to $375 \mathrm{mg}$ twice daily. Eight weeks later, his best corrected visual acuity was $6 / 6$ in both eyes. Fundoscopy showed a definite resolution of optic disc edema with spontaneous venous pulsations in both eyes (Figures 3(a) and 3(b)).

Visual field testing showed normal visual fields in both eyes. However, acetazolamide treatment ( $375 \mathrm{mg}$ daily) was continued for four further weeks and rhGH was not started up again. The total duration of acetazolamide treatment was 16 weeks.

\section{Discussion}

The association between rhGH and the development of PTC was first reported by Otten et al. in 1992 [13]. A progressive trend in higher and more frequent doses of growth hormone resulted in an increase of reported cases $[8,14,15]$. It is well known nowadays that the rhGH-induced PTC is doserelated [16]. It is also estimated that the incidence of rhGHinduced PTC is 1, 2 per 1000 cases among the patients receiving treatment with rhGH [4]. Malozowski and Koller reported that in most cases the dosage of rhGH ranged from 0.17 to $0.35 \mathrm{mg} / \mathrm{kg}$ per week and onset of symptoms occurred from 1 week to 5 years but usually within days $[1,17-$ 19]. In our case, symptoms occurred two months after the beginning of the treatment with rhGH on a standard dose of $1,5 \mathrm{mg}$ daily. The pathogenesis of rhGH-induced PTC may involve the increased cerebrospinal fluid (CSF) production by the choroid plexus. This production is secondary to an increase in growth hormone concentrations in the CSF which induce both the local production of IGF-1 and the activation of IGF-1 receptors. Alterations in sodium and water retention mediated by acute stimulation of the reninangiotensin system may also play an important role in the pathogenesis of the condition $[1,8,16]$. All children receiving rhGH should be monitored closely and have a complete investigation if they report headache or visual disturbances after the initiation of the treatment. Children with suspected PTC should have a careful neurologic and ophthalmologic evaluation including visual acuity assessment, color vision testing, papillary reflex evaluation, fundoscopic examination, 


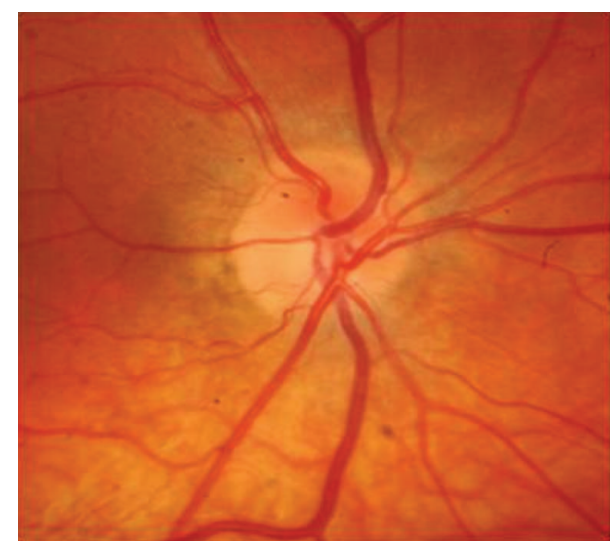

(a)

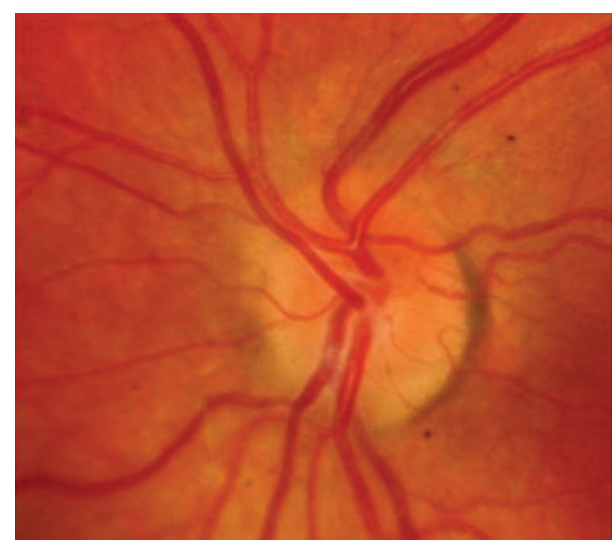

(b)

Figure 3: ((a), (b)) Definite right and left optic nerve edema resolution after 8 weeks.

and visual fields testing. Our patient was further investigated by an ophthalmologist and a neurologist and an rhGHinduced PTC was diagnosed. PTC usually goes into spontaneous remission. Indications for treatment are persistent headache or progressive visual deterioration due to optic nerve involvement [2]. The cornerstone of medical treatment is weight control for obese patients and cessation of any drug thought to have precipitated the condition [14]. The standard treatment includes diuretics and most notably acetazolamide [2]. In our case, the rhGH was discontinued and a treatment with acetazolamide per os was initiated shortly after a diagnosis of rhGH-induced PTC was established. In patients with severe deterioration of vision not responding adequately to medications, lumbar puncture (which could be used both diagnostically and therapeutically) and surgical procedures such as cerebrospinal fluid diversion procedure (lumboperitoneal or ventriculoperitoneal shunt) and optic nerve fenestration are recommended. In the majority of cases, resolution of both papilledema and symptoms of intracranial hypertension may take 3 to 6 months after discontinuation of rhGH. In our case, both signs and symptoms disappeared two months later. Our patient completed his treatment with decreasing doses of acetazolamide for sixteen weeks and the rhGH was not restarted. Restarting treatment on the same dose usually causes recurrence of the PTC which could be prevented if the treatment is restarted on a lower dose $[8,16]$. During the follow-up period all children should be evaluated by an ophthalmologist and a pediatric endocrinologist. In our case, the close collaboration between them resulted in rapid resolution of signs and symptoms and absolute recovery of visual acuity in both eyes.

\section{Conflict of Interests}

The authors declare that there is no conflict of interests regarding the publication of this paper.

\section{References}

[1] S. Malozowski, L. A. Tanner, D. Wysowski, and C. A. Fleming, "Growth hormone, insulin-like growth factor I, and benign intracranial hypertension," The New England Journal of Medicine, vol. 329, no. 9, pp. 665-666, 1993.

[2] S. Dhungana, B. Sharrack, and N. Woodroofe, "Idiopathic intracranial hypertension," Acta Neurologica Scandinavica, vol. 121, no. 2, pp. 71-82, 2010.

[3] M. Wall, J. J. Corbett, D. I. Friedman, G. Liu, and K. Digre, "Revised diagnostic criteria for the pseudotumor cerebri syndrome in adults and children," Neurology, vol. 83, no. 2, pp. 198200, 2014.

[4] P. Babikian, J. Corbett, and W. Bell, "Idiopathic intracranial hypertension in children: the Iowa experience," Journal of Child Neurology, vol. 9, no. 2, pp. 144-149, 1994.

[5] L. M. Rangwala and G. T. Liu, "Pediatric idiopathic intracranial hypertension," Survey of Ophthalmology, vol. 52, no. 6, pp. 597$617,2007$.

[6] S. Lessell, "Pediatric pseudotumor cerebri (idiopathic intracranial hypertension)," Survey of Ophthalmology, vol. 37, no. 3, pp. 155-166, 1992.

[7] P. H. Phillips, M. X. Repka, and S. R. Lambert, "Pseudotumor cerebri in children," Journal of AAPOS, vol. 2, no. 1, pp. 33-38, 1998.

[8] A. H. Rogers, G. L. Rogers, D. L. Bremer, and M. L. McGregor, "Pseudotumor cerebri in children receiving recombinant human growth hormone," Ophthalmology, vol. 106, no. 6, pp. 1186-1190, 1999.

[9] G. Tornese, G. Tonini, F. Patarino, F. Parentin, and F. Marchetti, "Double adverse drug reaction: recombinant human growth hormone and idiopathic intracranial hypertensionacetazolamide and metabolic acidosis: a case report," Cases Journal, vol. 2, article 6534, 2009.

[10] G. D. Reeves and D. A. Doyle, "Growth hormone treatment and pseudotumor cerebri: coincidence or close relationship?" Journal of Pediatric Endocrinology and Metabolism, vol. 15, supplement 2, pp. 723-730, 2002.

[11] P. E. Clayton and C. T. Cowell, "Safety issues in children and adolescents during growth hormone therapy - a review," Growth Hormone and IGF Research, vol. 10, no. 6, pp. 306-317, 2000.

[12] L. Frisen, "Swelling of the optic nerve head: a staging scheme," Journal of Neurology, Neurosurgery \& Psychiatry, vol. 45, no. 1, pp. 13-18, 1982. 
[13] B. J. Otten, J. J. Roettvel, and J. R. M. Cruysberg, "Pseudotumor cerebri following treatment with growth hormone," Hormone Research, vol. 37, p. 16, 1992.

[14] S. Bechtold, O. Butenandt, A. Meidert, H. Schmidt, and K.-P. Boergen, "Persistent papilledema in Ullrich-Turner syndrome treated with growth hormone," Clinical Pediatrics, vol. 40, no. 11, pp. 629-631, 2001.

[15] F. Distelmaier, U. Sengler, M. Messing-Juenger, B. Assmann, E. Mayatepek, and T. Rosenbaum, "Pseudotumor cerebri as an important differential diagnosis of papilledema in children," Brain and Development, vol. 28, no. 3, pp. 190-195, 2006.

[16] I. Francois, I. Casteels, J. Silberstein, P. Casaer, and F. De Zegher, "Empty sella, growth hormone deficiency and pseudotumour cerebri: effect of initiation, withdrawal and resumption of growth hormone therapy," European Journal of Pediatrics, vol. 156, no. 1, pp. 69-70, 1997.

[17] P. A. Crock, J. D. McKenzie, A. M. Nicoll et al., "Benign intracranial hypertension and recombinant growth hormone therapy in Australia and New Zealand," Acta Paediatrica, vol. 87, no. 4, pp. 381-386, 1998.

[18] E. A. Koller, B. V. Stadel, and S. N. Malozowski, "Papilledema in 15 renally compromised patients treated with growth hormone," Pediatric Nephrology, vol. 11, no. 4, pp. 451-454, 1997.

[19] D. A. Price, P. E. Clayton, and I. C. Lloyd, "Benign intracranial hypertension induced by growth hormone treatment," The Lancet, vol. 345, no. 8947, pp. 458-459, 1995. 




The Scientific World Journal


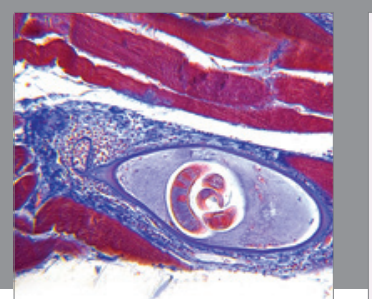

Gastroenterology Research and Practice

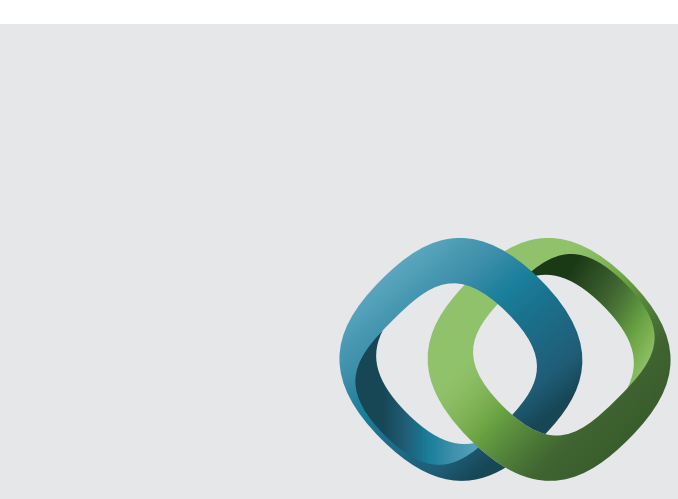

\section{Hindawi}

Submit your manuscripts at

http://www.hindawi.com
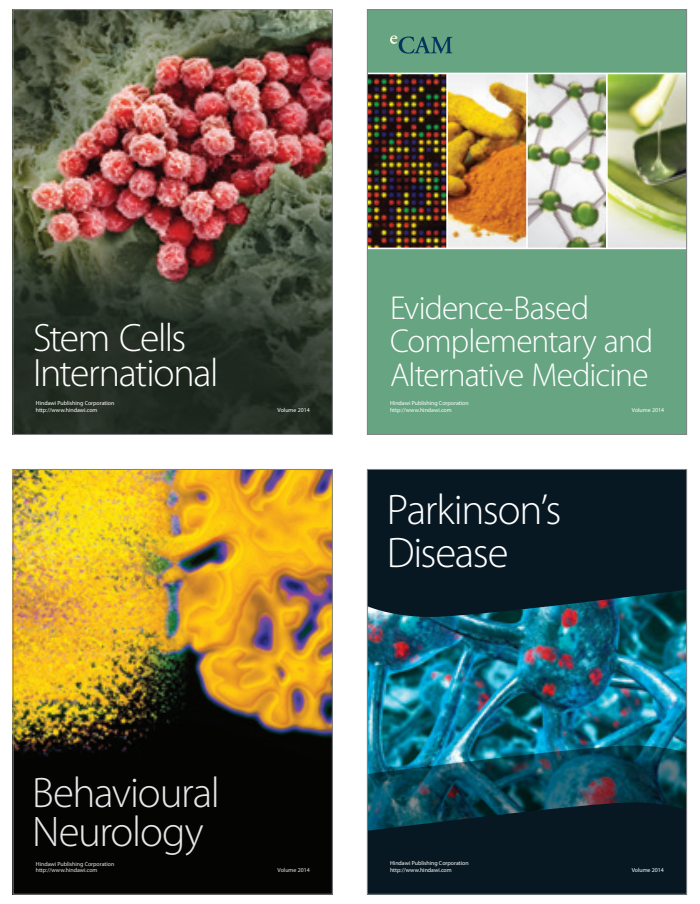
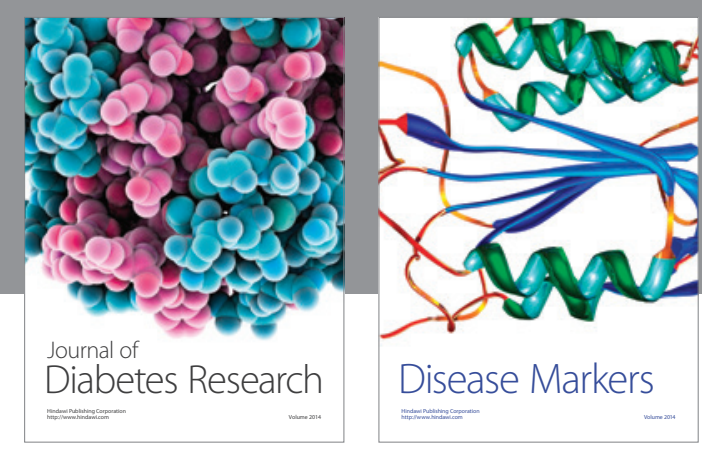

Disease Markers
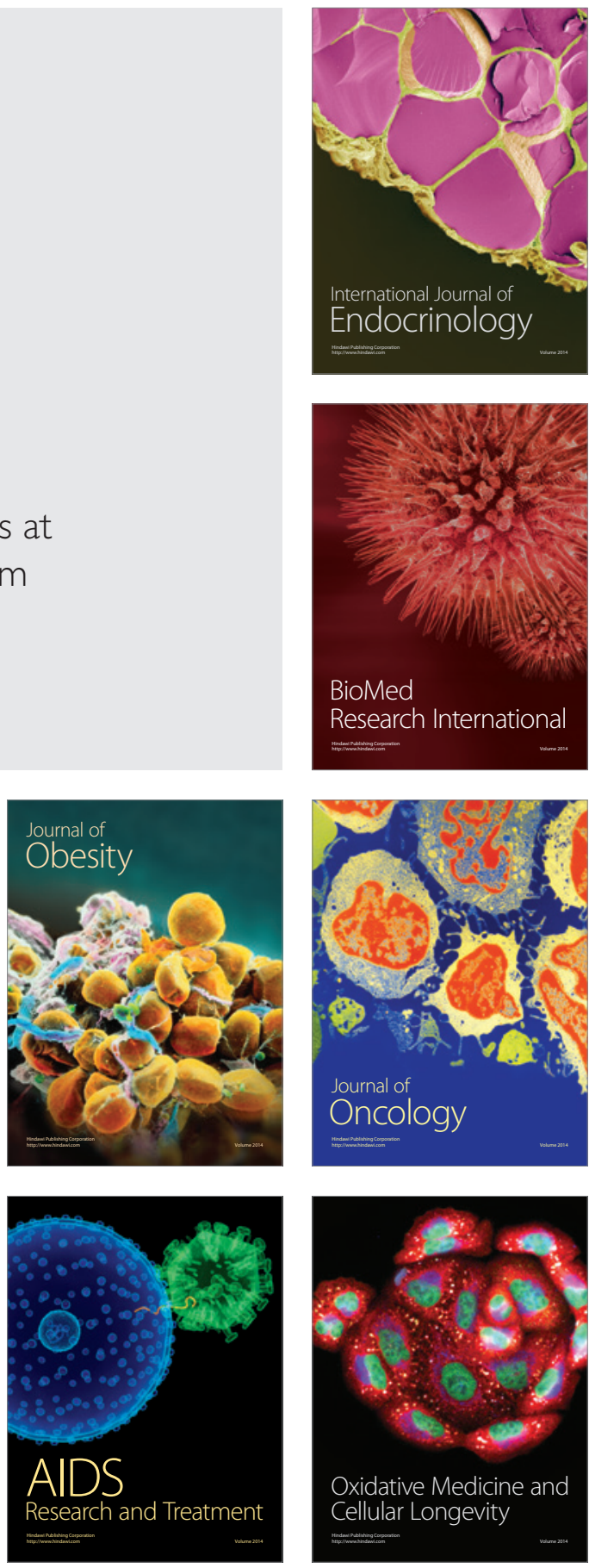